\title{
Supervisión y acompañamiento de prácticas profesionales. Desarrollo de un enfoque de reflexividad práctica desde un self-study colaborativo *
}

\author{
Solange Gorichon Gálvez ${ }^{1}$ (D) @ \\ Alicia Pérez Lorca ${ }^{2}$ (D) @ \\ Mariluz Yañez Pérez ${ }^{1}$ (1) @ \\ Mabel Roberts Rivera ${ }^{2}$ (D) @ \\ ${ }^{1}$ Universidad Alberto Hurtado, Chile; ${ }^{2}$ Universidad de Santiago de Chile (USACH), Chile.
}

Resumen. Este artículo recoge el proceso de indagación colectivo y sistemático de cuatro académicas, en relación a la enseñanza de la reflexión en el contexto de prácticas profesionales de estudiantes de pedagogía implementado con metodología self-study. Sus resultados manifiestan las tensiones profesionales suscitadas de la revisión de nuestras prácticas de enseñanza, la relevancia de las propias concepciones sobre el rol docente y la importancia de la comunidad de amigos críticos en el proceso reflexivo para poner en valor los dilemas y aprendizajes de un ejercicio profesional muchas veces solitario y poco estudiado. En ese contexto, el self-study busca ser tanto un enfoque de investigación de la propia práctica en académicos universitarios, como una instancia para mejorar las prácticas de enseñanza a través de la generación de conocimientos prácticos de la profesión con el fin de posibilitar mejores aprendizajes en los estudiantes de pedagogía.

Palabras clave: self-study, formación inicial docente, prácticum, reflexión docente, comunidad de aprendizaje.

Supervisão e acompanhamento de estágios profissionais. Desenvolvimento de um enfoque de
prática reflexiva a partir de um self-study colaborativo
Resumo. Este artigo apresenta o processo de questionamento coletivo e sistemático de quatro
universitárias, relacionado com o ensino da reflexão no contexto de estágios profissionais de
estudantes de pedagogia, implementado com a metodologia do autoestudo (self-study). Os seus
resultados evidenciam as tensões profissionais geradas pela revisão das nossas práticas de ensino,
a relevância das próprias concepções sobre o papel docente e a importância da comunidade de
amigos críticos no processo de reflexão para ressaltar os dilemas e aprendizagens de um exercício
profissional muitas vezes solitário e pouco estudado. Neste contexto, o self-study pretende ser tanto
uma abordagem de pesquisa da própria prática de universitários, quanto um recurso para melhorar
os estágios através da geração de conhecimentos práticos da profissão, possibilitando melhores
aprendizagens nos estudantes de pedagogia. Palavras-chave: self-study, autoestudo, formação inicial docente, estágio, reflexão docente, comunidade de aprendizagem.

Supervision and accompaniment of professional practices. Development of a practical reflexivity approach from a collaborative self-study

Abstract. This article gathers the process of collective and systematic inquiry of four lecturers employing the self-study methodology in connection with the teaching of reflection during the teacher training practicum process of pedagogy students. The results show the professional tensions raised in the review of the teaching practicum, the relevance of the participants' own conceptions about the teaching role and the importance of critical friends' community in the reflective process. This contributed to the value of the dilemmas and learning of a professional exercise that is often lonely and poorly studied. In this context, the proposed self-study seeks to be both a research approach of the practice itself in lecturers and an instance to improve teaching practices through the generation of practical knowledge in order to enable better learning in pedagogy students.

Keywords: self-study, teacher training, practicum, teacher reflection, learning community.

* Esta investigación es fruto del proyecto "Self-Study colaborativo sobre prácticas de formación docente" (2016-2017), financiada por la Organización de Estados Iberoamericanos. 


\section{Introducción}

La experiencia como maestras de escuela y académicas universitarias para la formación del profesorado en Chile, nos ha permitido experimentar de cerca las dificultades que tienen estos para insertarse en el mundo laboral. La escuela como lugar de trabajo y de formación, se constituye en un espacio altamente complejo, donde la rigurosa aplicación técnica de saberes no logra dar respuesta a las situaciones que allí se enfrentan. La profesión docente requiere, asimismo, de una constante revisión de las prácticas y las situaciones pedagógicas con el fin de ayudar a la transformación y mejora de los procesos educativos. De allí la importancia de la generación de espacios que permitan la reflexión de dichos procesos, especialmente para aquellos y aquellas que se están formando para ser profesores.

En la actualidad, variados autores consideran que el ejercicio reflexivo que realizan los futuros docentes sobre la propia práctica de enseñanza -a la luz de los saberes didáctico, disciplinar y pedagógico- puede contribuir a explicar sus propias acciones docentes. Tal como lo expresa Castellanos y Yaya (2013), los conocimientos de los cuales se apropian los futuros docentes para explicar y problematizar su enseñanza son un recurso que les permite ampliar la mirada sobre sus acciones y conceptualizar su propio quehacer. Lo anterior requiere que el estudiantado sea formado en un hábito de reflexión y análisis de la propia práctica docente, que se prolongue durante toda su vida laboral y profesional (Guerra, 2009).

Conscientes de aquello, y de las tensiones que teníamos cada una respecto de la formación que estábamos dando a nuestros estudiantes, es que decidimos realizar un proceso que nos permitiera mirar nuestra práctica. Desde hace algunos años, trabajamos en acompañamiento de prácticas intermedia y final, promoviendo la competencia reflexiva mediante el análisis de incidentes críticos y discusión de propuestas pedagógicas. Sin embargo, porque conocemos la complejidad de los centros educativos y sus demandas, no tan solo esperamos formar futuros docentes de excelencia profesional, sino también comprometidos con su rol social. Nos preocupaba poder brindar una formación que interpelara a los futuros profesionales no sólo acerca de su enseñanza, sino también acerca de la sociedad en la que viven y la importancia de su preparación para el logro de aprendizajes relevantes en los niños y jóvenes de Chile.

En ese sentido, ¿cómo podíamos mejorar nuestra labor de acompañamiento y supervisión de prácticas a estudiantes de pedagogía para el desarrollo de una competencia reflexiva? Esta amplia pregunta derivó en las siguientes interrogantes: 
- ¿Qué finalidades tiene el desarrollo de una competencia reflexiva?

- ¿Qué docente queremos formar en el espacio de práctica profesional?

- ¿Sobre qué debemos hacerles reflexionar?

- ¿Qué acciones de acompañamiento impactan favorablemente el desarrollo de la competencia reflexiva en mis estudiantes?

La experiencia de reflexión conjunta fue fundamental para sensibilizarnos, elaborar nuevos conocimientos sobre la práctica y abrirnos a la posibilidad de transformar los procesos de enseñanza y aprendizaje que llevamos a cabo en nuestras aulas universitarias, conscientes de que el análisis de la práctica es en gran parte un trabajo personal, para este escrito tomamos la decisión de narrar el proceso colectivo que implicó el acompañamiento mutuo frente a los dilemas que conjuntamente fuimos definiendo.

A continuación, se narra el proceso de self-study conformado por los dilemas surgidos en el trayecto de observar nuestro quehacer y ponerlo en diálogo con la comunidad de amigas críticas.

\section{La metodología que escogimos}

\section{a) Quiénes somos y cómo nos organizamos}

Somos cuatro profesoras que realizamos docencia en carreras de pedagogía en distintas universidades de Santiago de Chile. Las asignaturas que dictamos se relacionan con la práctica profesional, tanto en espacios de acompañamiento periódico y supervisión de prácticas ( 2 a 4 estudiantes), como en modalidad de taller semanal de análisis de práctica ( 15 a 40 estudiantes).

Considerando que el trabajo personal, la autoevaluación, la autocrítica y la apertura al cambio son consideradas condiciones esenciales para este self-study (Cornejo, 2016), como de igual modo, contar con un amigo crítico que ayude en el proceso de autoconocimiento (Schuck \& Russell, 2016); es que barajamos primeramente cuatro modalidades de colaboración (figura 1).

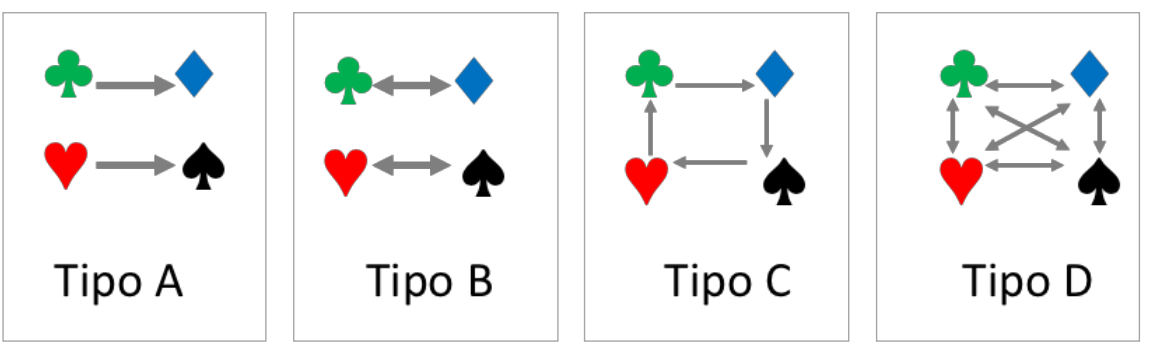

Figura 1: Formas de organizar el self-study colaborativo (elaboración propia) 
Inicialmente, la opción A nos parecía una forma ordenada de estructurar la amistad crítica, donde cada participante asumía un rol único, es decir como amiga crítica o bien como investigadora. Sin embargo, al igual que la opción C, consideramos perdíamos la experiencia profesional de cada una. Por otra parte, la opción B nos daba la posibilidad de interactuar en un doble rol, pero perdiendo las ventajas de un trabajo interuniversitario. Finalmente, optamos por la modalidad $\mathrm{D}$, por ser aquella que consideramos más rica en cuanto a interacciones, aprovechando que cada una de nosotras investigaría su propia práctica y actuaría al mismo tiempo como amiga crítica.

En este contexto, entendemos la colaboración como una oportunidad para construir significados conjuntamente, mediante el intercambio de experiencias y comprensiones sobre el fenómeno educativo, como también, el trabajo conjunto de un grupo de profesores, utilizando distintas modalidades que permitan mejorar la competencia profesional y garantizar el crecimiento de todos (Blanch \& Corcelles, 2014). Tal como lo expresa Moreno (2006), la cultura de colaboración entre docentes es considerada hoy en día un elemento indispensable para la innovación educativa.

Se potenció la creación de una comunidad profesional de aprendizaje, resguardando un clima de confianza entre aquellas que queríamos aventurarnos en este viaje, promoviendo en todo momento la escucha atenta y amorosa respecto del trabajo de la otra, con gran disposición para contribuir en la mejora de las prácticas de las demás (Schuck \& Russell, 2016).

Las reuniones se realizaron semanalmente para mantener cierta frecuencia en nuestros encuentros y fortalecer el lazo que estábamos creando. Valoramos la posibilidad de reflexionar de manera conjunta periódicamente, tanto para mantener cierto ritmo de trabajo, como para poder compartir de manera oportuna las reflexiones, dificultades y cuestionamientos que iban emergiendo en la práctica de cada una.

Cada sesión de trabajo fue organizada sobre incidentes críticos (Monereo, 2014) que gatillaban distintos cuestionamientos a nivel personal como grupal. Estos fueron derivados de la lectura y análisis de las bitácoras, como del diálogo conjunto.

El rol de las amigas críticas se centró, por tanto, en tensionar a cada integrante de la comunidad sobre las decisiones tomadas en el proceso y cómo estas potenciaron o no, el desarrollo de una competencia reflexiva en torno a la propia práctica docente. Se utilizó de manera inconsciente en el inicio y conscientemente luego, la argumentación didáctica como mecanismo para promover la propia reflexión (Fenstermacher, 1987). 
Cautelamos que las reuniones se iniciaran a partir de narrativas de nuestras prácticas, las que iríamos rotando en las distintas semanas con el fin de prever que todas tuviésemos el espacio de compartir situaciones y reflexiones suscitadas en la práctica.

\section{b) Las fuentes de evidencia}

El proceso de analizar la propia práctica como formadoras implicó observar las historias de vida que conformaron nuestra identidad profesional. Por ello, se recurrió a tres fuentes principales de información: las reflexiones personales, el trabajo y percepción del estudiantado y la mirada de las amigas críticas (figura 2). Cada uno de estos elementos fue nutrido a partir de dispositivos específicos que señalaremos a continuación.

\section{El proceso de mirar la práctica como formadoras}

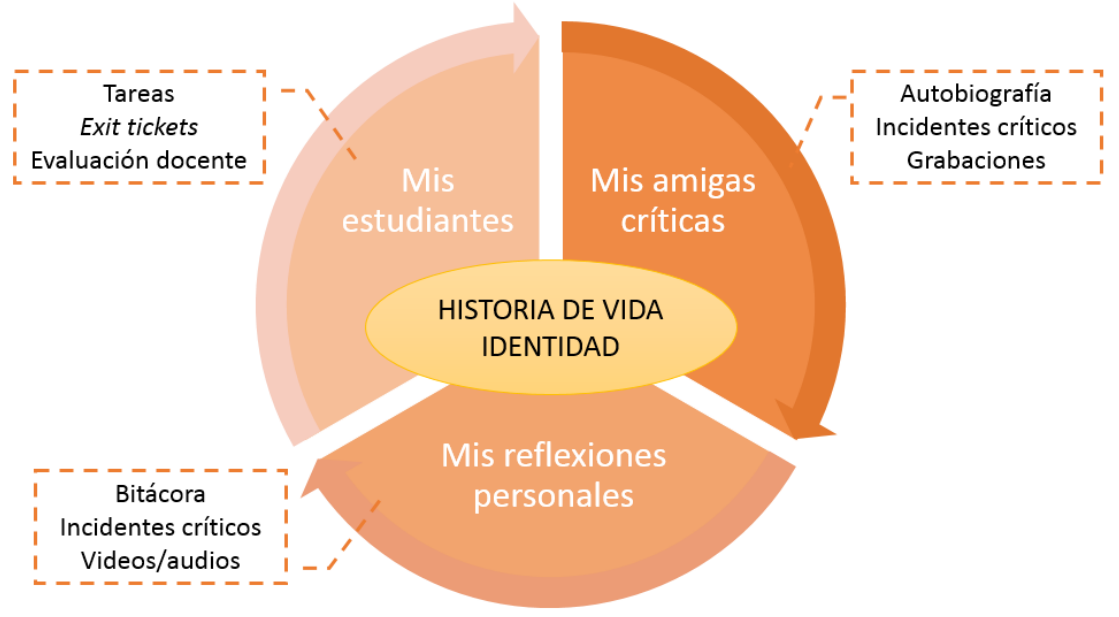

Figura 2. Dispositivos y espacios para mirar la propia práctica a través del self-study

Primeramente, se pidió que cada participante considera las tareas y actividades realizadas por sus estudiantes, así como los diálogos referidos a la experiencia de aprendizaje en su curso. Se solicitó en particular llevar registro del trabajo con estudiantes en formato de audio o video (al menos dos entradas) para poder observar el desarrollo de la acción docente con ellos: las formas de acompañamiento, las estrategias para orientar la reflexión pedagógica y el tipo de discurso instalado en estos espacios de aprendizaje. Cabe señalar que el registro del trabajo de los estudiantes no fue analizado en esta investigación, sino que fue utilizado para estimular la reflexión personal en el proceso, el que posteriormente se traduciría en un insumo vital para la reflexión colectiva y posteriores mejoras que realizamos en nuestras prácticas de acompañamiento. 
A partir de lo anterior, se desarrolló una bitácora personal con el registro semanal de cada una de las actividades docentes realizadas. En ella, se identificó los incidentes críticos percibidos, lo cual sirvió de insumo para las discusiones semanales en el grupo.

En tercer lugar y al inicio de la investigación, se solicitó una autobiografía breve (2 a 3 páginas) acerca de cómo se configuró nuestra identidad profesional y los motivos que impulsaban nuestro quehacer docente. El objetivo era poder conocer la trayectoria profesional de cada una e iniciar la reflexión personal a través del reconocimiento los propios marcos interpretativos.

De esta manera, el self-study sobre nuestra propia práctica docente como formadoras de formadores, consideró tanto los insumos que el trabajo cotidiano con nuestros alumnos nos proveía, como los dispositivos específicos elaborados para este proceso (autobiografía y bitácora) y las preguntas, tensiones y reflexiones emanadas del trabajo colaborativo con las amigas críticas.

\section{c) El análisis}

El análisis de este estudio se realizó en base a las grabaciones de audio de 11 sesiones de trabajo realizadas entre octubre 2016 y mayo 2017. Las grabaciones fueron transcritas y analizadas por medio de Atlas-Ti.

Considerando que cada sesión de trabajo contenía uno o más incidentes críticos para la reflexión, se puso especial cuidado en identificar los detonadores que facilitaron o gatillaron los focos de preocupación e indagación de manera individual como grupal y que permitieron cuestionar la acción pedagógica ejercida como formadoras y los supuestos detrás de ellos.

También se consideró la recursividad con que surgían las tensiones, en algunos casos se matizaban y/o volvían a resurgir en las conversaciones en la comunidad de amigas críticas o se seguían profundizando en las reflexiones individuales.

\section{Nuestras tensiones y dilemas surgidos en el self-study}

Considerando que el punto de partida de este self-studyera indagar respecto de los elementos de la propia práctica que favorecían el desarrollo de una competencia reflexiva en nuestros estudiantes, se explicitaron las formas o maneras en que gestionábamos el proceso de enseñanza y aprendizaje en el contexto de las prácticas profesionales. 
Para orientar el análisis, iniciaremos con una breve caracterización de las tensiones que nos movilizaban individualmente al momento de comenzar este self-study. Los cuatro puntos de partida fueron:

La enseñanza de las Ciencias Sociales: Formando a futuros profesores de secundaria, sentía la necesidad de ayudarles a enfrentar los dilemas específicos de la enseñanza de las ciencias sociales. La tutoría de práctica se convertía en un espacio de reflexión sobre los problemas de la escuela -la conducta de las y los alumnos, la relación con sus pares y apoderados-, pero costaba mucho más profundizar en aquello que sólo ellos debían saber enseñar: pensamiento histórico y social. Comencé a preguntarme cómo estaba ayudándoles a reflexionar y de qué manera podía hacerlo mejor (Alicia).

Desnaturalizar los supuestos: Las formas de "enseñar" y "aprender" que experimentamos en nuestra trayectoria formativa escolar y universitaria marcaron nuestros supuestos acerca de cómo se debe enseñar y aprender. En mis talleres de reflexión sobre la práctica me preguntaba cómo movilizar los supuestos de mis estudiantes hacia enfoques más críticos y reflexivos de nuestro quehacer pedagógico en las aulas, en un contexto de valoración de racionalidades técnicas y academicistas. Comencé a preguntarme cómo ayudar a desnaturalizar las propias prácticas de enseñanza y formas de relacionarnos con los estudiantes, como también, ser críticos de las prácticas que observamos en el sistema escolar. Había que comenzar por mirar lo que estaba haciendo con otros, pues sentía que estaba teniendo una mirada muy complaciente de mis prácticas en los talleres que impartía. El self-study me daba una gran oportunidad para ello (Solange).

Transitar de lo técnico a lo crítico: Desde el año 2013 nuestra carrera estaba enfrentando un cambio de malla profundo y entre otros desafíos estábamos llamados a formar profesores capaces de reflexionar con un sentido crítico, entonces se hacía necesario mirarnos y ver en qué medida cumplíamos con este objetivo en el contexto de los talleres de reflexión ligados a la práctica profesional y así comenzamos a preguntarnos en qué consiste la reflexión crítica; cómo podemos ir más allá de la reflexión técnica que realizábamos habitualmente; hasta qué punto es legítimo proponer un desarrollo profesional basado en creencias o supuestos que no estaban consensuados y que dependían del profesional que guiara los talleres. Se hacía muy necesario develar los supuestos que guiaban nuestra reflexión e intencionar procesos que permitieran el desarrollo de profesionales reflexivos críticos. La investigación colaborativa era clave para reconocernos (Mabel).

La tarea del tutor entre lo objetivo y lo subjetivo: En mi carrera hay dos pilares fundamentales en la formación de profesores; la formación práctica y la reflexión. Para ello hemos elaborado programas que promueven el desarrollo de la reflexividad a partir de experiencias auténticas en la escuela. En la retroalimentación dada a los estudiantes se realiza un diálogo sobre sus desempeños, es en estos espacios que me surge la pregunta respecto ¿cuál es el tipo de apoyo que debo proporcionarles para que sean buenos 
profesores? Es en este escenario de tutoría en donde se evidencia mis representaciones sobre buenas prácticas y reflexividad. Pensé que a partir de la exposición en un espacio de confianza podía mirarme y tomar conciencia del impacto que tiene en mi quehacer de formadora de formadores esta dimensión subjetiva de mi quehacer (Mariluz).

Las cuatro inquietudes convergieron en la siguiente pregunta que nos movilizó: ¿cómo mejorar nuestra labor de acompañamiento y supervisión de prácticas a estudiantes de pedagogía para el desarrollo de una competencia reflexiva?

Para responder a dicha tensión inicial, organizamos el análisis en las siguientes preocupaciones: para qué enseñar a reflexionar, qué docente queremos formar, sobre qué propender en la reflexión y qué acciones de acompañamiento impactan el desarrollo de la competencia reflexiva en los estudiantes.

\section{a) ¿Para qué enseñar a reflexionar?: comprender la práctica situa- da $\mathrm{v} / \mathrm{s}$ transformar la sociedad}

Al inicio observamos la gran diversidad de propósitos que le asignábamos a la reflexión y, con ello, que las definiciones de reflexión y competencia reflexiva no estaban claras. Primeramente, discutimos acerca de lo que entendíamos por reflexión:

Es un hábito intelectual, yo creo que la reflexión como otras actividades cognitivas es un hábito. Entonces tal vez la clave está en cómo hacemos esto en un proceso, en un continuo y no es algo que va a pasar al final de la carrera, sino que va a pasar durante todo el proceso formativo. Hay distintas experiencias que van a hacer que esto sea un proceso antes de constituir un hábito (Mariluz).

¿Qué estamos entendiendo por reflexión? ¿es hacer un buen análisis? ¿es para la acción? [...] Que también eso deberíamos tenerlo súper claro en el momento que estamos intencionándolo, trabajándolo o construyéndolo con los estudiantes (Solange).

Sin tener del todo claridad acerca de una definición de competencia reflexiva, coincidimos en la complejidad de la misma: en ocasiones podía representarse como un producto acabado (e.g. reflexión crítica) o como un proceso en sí mismo (e.g. hábito). Sin embargo, si no teníamos claridad de su definición, ¿por qué seguíamos formando en la reflexión?

Por una parte, coincidimos en su relevancia para mejorar la práctica docente a partir del análisis de las prácticas situadas: 
[...] es que hay investigación que reafirma que el profesional es mejor en la medida que reflexiona sobre su quehacer. Por lo tanto, nos permite justificar que la acción técnica rigurosa, no es suficiente para poder desempeñarte bien profesionalmente. Y esa cuestión a mí me encanta, poder decir 'no es un tema mío personal' [sino] que el profesional tiene limitaciones si no es capaz de reflexionar analíticamente, mirar su práctica en base a ciertos criterios, porque nada que haga en la universidad lo va a preparar para las miles de problemáticas que va a enfrentar [...] (Alicia).

Sin embargo, también discutimos que la reflexión debía permitir, más que resolver problemas del quehacer profesional, involucrar a los futuros docentes en la transformación de la sociedad en que viven:

Yo espero una reflexión crítica, que poco más los estudiantes cambien el mundo... asi partí, pero a partir de otro estudio que estaba leyendo acerca de qué es la reflexión, he cambiado mi percepción: hay reflexiones que son súper analíticas, y eso es un proceso súper cognitivo...y no toda reflexión implica transformación y cambio (Solange).

Al realizar el análisis posterior de nuestros encuentros, pudimos notar que veíamos cuatro grandes finalidades de la reflexión: i) como una manera de comprender y fundamentar su quehacer; ii) como una manera de impactar en la escuela a través de la confrontación de sus decisiones con otros; y iii) como una forma de cuestionar su propio rol profesional.

Concluimos que era relevante tener orientaciones curriculares acerca de qué se esperaba de la reflexión, y con ello, los alcances de la misma. Fuimos conscientes de que la reflexión es una competencia profesional que se desarrolla a lo largo de la vida y por ello, definir un propósito nos ayudaría a orientar mejor las acciones formativas en el espacio de práctica, que es por lo demás acotado y finito.

Por supuesto, clarificar esta tensión generó nuevas interrogantes, como por ejemplo ¿cuál era el papel de la práctica profesional y el rol profesional docente?

\section{b) ¿Qué docente queremos formar?: Imponer concepciones v/s} favorecer la construcción de la propia identidad docente

Sin tanta claridad al inicio respecto de qué es reflexionar para cada una de nosotras y sobre la finalidad de formar una competencia reflexiva, discutimos ampliamente respecto de cómo queríamos que fuese el y la docente que formáramos.

Observamos en todas nosotras la tendencia a concebir la responsabilidad de la profesión desde su capacidad para transformar la sociedad: 
La creencia en la base, es tener un profesor comprometido con su comunidad y el desarrollo social, y nos damos cuenta de que algunos estudiantes quieren ganar dinero, entre otras cosas. Entonces ¿cómo entender y aceptar al otro? (Mabel).

Desde esta concepción, notamos a lo largo del estudio que teníamos un juicio sobre la forma en que nuestros estudiantes se vinculaban con el mundo y con la profesión docente:

Nosotros tenemos estudiantes que hacen el "check-list": "entregué mi planificación con 4 días de anticipación, en la clase motivé a los alumnos, mis alumnos guardan silencio' [...] Pero cuando es el aniversario del colegio no se quieren involucrar, hay reunión de departamento y no van... Hay una actitud -no todos-, pero viene un grupo que es así [...] por eso yo estoy pensando en algunos estudiantes, en lo actitudinal. Hay generaciones maravillosas [pero otras] no quieren comprometerse (Mabel).

Estamos en un modelo en el cual tener una profesión es una cuestión instrumental. No tiene que ver con un desarrollo personal o desarrollo humano [...] La educación te prepara para el mundo del trabajo. Entonces, cuando tenemos chiquillos así, es súper difícil, porque es contracultural (Mariluz).

Nos dimos cuenta que nuestras expectativas eran mayores, pues no bastaba con el análisis crítico sobre la práctica, sino que esperábamos que se convirtieran en actores sociales involucrados en la promoción y desarrollo de las personas, lo cual implica un rol transformador de la sociedad:

Mira la tensión que le estamos provocando [al estudiante], primero le estamos modelando lo que nosotras pensamos, nuestras propias concepciones, pero además lo estamos tensionando a que las concepciones que deben tener son las nuestras (Mabel).

En el proceso tomamos consciencia cómo influía el peso de nuestra historia en la forma de orientar las sesiones de acompañamiento con nuestros estudiantes. Esto implicó reconocer que nuestra mirada de la sociedad no podía obligar a los estudiantes a conformar su ser docente. Por el contrario, la reflexión debía permitirles encontrar sus propias formas de leer el rol de agente social que tiene la profesión docente en este momento.

También vislumbramos que la forma de entender la profesión se relacionó fuertemente a la comprensión de nuestro propio rol como profesoras. Una de las concepciones más profundas era la intención de salvar a nuestros estudiantes de una serie de dificultades que presenta la escuela -o incluso de sus propias realidades- $\mathrm{y}$ con ello, tensionamos permanentemente el espacio de formación de la práctica. Le denominamos "rol mesiánico" pues sentíamos que era el sentido de una docencia salvadora. Este rol nos obligaba muchas 
veces a adquirir funciones mucho más allá de nuestro trabajo como profesoras supervisoras/tutoras; pero no tan solo eso, nos dimos cuenta de que también era parte de lo que les exigíamos a nuestro estudiantado:

Somos bastante mesiánicas, hay una fuerza en nosotras que nos empuja a querer salvar y en ese salvar pensamos que la reflexión debía tener ciertas características, pero aquellas no necesariamente ayudan a los estudiantes a desarrollar estas características que consideramos tan importantes(Mariluz).

La escritura y análisis de la autobiografía nos reveló la importancia de nuestra propia historia en la configuración de nuestro rol de tutoras, pues nuestra visión acerca de la educación condicionaba las exigencias y expectativas sobre nuestros/as estudiantes. Indagar sobre nuestras historias de vida, nos hizo reconocer la relevancia de nuestras creencias y concepciones a la hora de realizar el acompañamiento y supervisión de prácticas.

Es decir, antes de pensar en mejorar nuestras prácticas de enseñanza de la reflexión, fue fundamental identificar desde dónde miramos nuestro trabajo y cuál es el rol que le compete a las y los futuros docentes. Temimos muchas veces que nuestras reflexiones se convirtieran en el estándar exigido a nuestro estudiantado, obligándolos a ser como nosotras, pero aprendimos que debíamos ser cuidadosas en el proceso de acompañamiento para fomentar la autonomía en la construcción de su propio rol profesional.

c) ¿Sobre qué reflexionar con el estudiantado? Problemáticas de su enseñanza situada v/s ayudarlos a conformar su identidad profesional

Al iniciar nuestro self-study fue importante clarificar cuáles eran los espacios de formación práctica en el que nos desempeñábamos y quiénes eran los estudiantes con que trabajábamos. En algunas oportunidades se nos cruzó el propósito de la reflexión con el sentido de la formación práctica en sí misma, por lo que no era extraño que hablásemos de las necesidades de nuestros alumnos cuando pensábamos sobre qué reflexionar.

En la sesión 5 una de las amigas críticas inicia la conversación con un dilema suscitado de su propia práctica:

[en el taller] estamos en los factores que inciden en la enseñanza y nos detuvimos en el profesor. Todo esto relacionado con las situaciones duras que les ha tocado vivir a ellos en los colegios y qué les pasa a ellos con esa experiencia. Entonces hay una serie de emociones que están ahí: sentimiento de decepción muchas veces, porque en contextos -sobre todo- de pobreza, sienten que la profesora es el único actor que puede hacer un cambio. Desesperanza, porque no se ven otras alternativas (a corto y mediano plazo) y es la vida de estos niños, un niño que está siendo 
vulnerado, que no está aprendiendo [...]. Como hacen mucho juicio sobre los profesores, [les pregunto] qué creen que sienten los profesores ante esta realidad. $Y$ comenzaron a ponerse en el lugar de los profesores y que esos sentimientos tal vez son similares, pero tratan de hacer algo, intentar algunas acciones que les permitan a estos niños tener algunas herramientas. Después, por algún momento -y no sé cómo llegamos ahí-, comenzamos a hablar de las emociones que nos empujan y fue bien preocupante para mí. Algunos me dijeron 'profesora, voy a pensar en la emoción o sentimiento que tengo permanentemente' y para otros, era un sentimiento de tristeza [...]. Es como una tristeza vital y me dio mucha vuelta (Mariluz).

Pensamos que la reflexión no sólo debía ayudarles técnicamente a resolver problemas de la enseñanza, sino también a fortalecer su inteligencia emocional. Ello implica hacerse cargo de lo que a cada uno le pasa en estos contextos, reconocer sus emociones y sentimientos y cómo abordarlos. Eso significa que existen dificultades de la práctica docente que no podemos resolver con "tips", sino que deben ser abordados reflexivamente respecto del propio actuar.

Comprendimos que el quehacer profesional tensiona a los estudiantes en elementos que muchas veces se ligan a su historia personal y eso les hace proyectar otros elementos en su rol como profesionales, tal cual como nos ocurría a nosotras. Con ello, fuimos conscientes de que nuestra preocupación por el bienestar de nuestros estudiantes pasaba, entre otras cosas, por tener la tranquilidad de que podríamos proporcionarles herramientas para sostener emocionalmente las complejidades que les deparaba el quehacer docente. Ello implicó reconocer que en las tutorías había que proporcionarles también espacios de contención, pues es, finalmente, en los espacios de práctica, donde se movilizan distintos tipos de disposiciones y capacidades que condicionan sus acciones pedagógicas

En este contexto, la definición de las problemáticas de la reflexión también fue tensionado a partir de las características de cada espacio de práctica y el rol que allí desempeñábamos. Para algunas, la reflexión sobre la práctica debía recoger las problemáticas de enseñanza en general y, para otras, estaba mucho más enfocada a la enseñanza de la disciplina o la reflexión sobre problemáticas puntuales para el aprendizaje de una materia. No llegamos a consensuar este aspecto, pero sí confluimos que el propósito de la reflexión en el espacio de formación práctica, está muy ligado a ayudarles a descubrirse y a "convertirse en docente", un espacio para aprender y trabajar su propia autonomía, desde el ejercicio permanente del auto-cuestionamiento de su práctica, el descubrimiento de sus propias fortalezas y dificultades en el desempeño, en el asumir el error como un espacio para aprender. 
Concluimos sobre la importancia de definir con mayor precisión a nivel de las carreras qué grado de competencia reflexiva se espera de un egresado, reconociendo incluso cómo a veces os docentes con años de experiencia tienen dificultad para alcanzar altos niveles de reflexión, así como también la necesidad de definir el foco de las reflexiones y los dispositivos necesarios para apoyarlos en este proceso de "convertirse en profesor o profesora", contemplando siempre sus contextos y la influencia de sus historias de vida en el desarrollo de su propia identidad docente.

d) ¿Qué acciones de acompañamiento impactan el desarrollo de la competencia reflexiva? ¿Existe una escucha, diálogo y apertura genuina?

En el inicio del self-study, cada vez que queríamos comentar sobre cómo gestionábamos el proceso de enseñanza y aprendizaje, nos quedábamos en la discusión de la finalidad y los propósitos. Sin embargo, a lo largo del proyecto fuimos notando algunos elementos que tenían mucho más valor de lo que pensábamos.

Notamos en el contexto de nuestro propio self-study la importancia del tipo de diálogo al interior del grupo y comenzamos a analizar cuáles habían sido los factores que habían facilitado nuestro propio proceso reflexivo:

Hemos instalado una nueva forma de mirar, hemos agregado un foco. Conservamos la mirada, pero hemos agregado uno nuevo. No cambia, pero se agrega algo [...] (Mabel).

[...] en el fondo fuimos reproduciendo o definiendo mecanismos que era lo que queríamos replicar con los chiquillos: esta lógica de ser cuidadosos, de generar un clima de confianza agradable a los sentidos (Alicia).

Consideramos que lo anterior requería de un clima especial de confianza, no solo para abrirse con un otro, sino también para poder realizar un diálogo honesto, en un marco de tiempo que favorezca el trabajo reflexivo:

Hay un elemento que me parece fundamental [...] por qué lo hago, cómo lo hago. Y ahí tiene que ver todo el tema de las creencias, de mi capital cultural, de mi experiencia vital. No sé si les pasa, pero yo tengo estudiantes que uno podría cuestionar por qué hacen lo que hacen. ¿cómo les cuestiono si no hay un espacio de confianza? Si yo necesito decirle 'tú me traes un certificado médico falso, porque no quieres ir hasta el último día de clases' (porque me ha pasado) [...] no basta con que se lo diga [...] Allí debe haber un proceso mayor de tomar consciencia de ese acto que influye sobre la vida escolar [...] Y este camino que tiene mucho bucles (Mariluz). 
Esta situación ilustra con claridad dos elementos. Por una parte, el reconocimiento de que la reflexión es un proceso progresivo, que a veces toma más tiempo que el que uno tiene planificado, como se refuerza a continuación:

La tensión que identifico es cómo jugamos con el tema de los tiempos, porque tenemos 1 hora para hacer el análisis [entonces] no puedo permitir - a partir de preguntas- que todo lo revele por sí mismo. Mi tensión es 'este es el minuto donde debo dar una clave adicional' (Alicia).

Y por otro lado, se hace evidente la importancia de una nueva perspectiva de escucha, que involucra una actitud genuina, abierta y disponible para acoger al otro. A partir de estas reflexiones, fuimos siendo más conscientes de los cambios que se iban suscitando en nuestras aulas.

Cuando empecé a escuchar a los chiquillos, atentamente, empáticamente, fueron saliendo temas y esos mismos temas era 'cómo le vamos poniendo marcos teóricos a esto', 'qué nos podría ayudar a entenderlo de mejor manera' (Mariluz).

Escucharlos genuinamente. Es esa la sensación, es como ‘despójate’ para poder escucharlos genuinamente (Solange).

Esta idea de la 'escucha genuina nos hizo tomar consciencia de la relevancia de escuchar más allá de lo estrictamente técnico en el desarrollo de una clase. Esto no siempre resultó sencillo, puesto que los estudiantes en formación poseen distintas motivaciones y una de estas es tener la acreditación de profesor para ejercer:

[es como que] por un lado, valoro a la persona que está enfrente -estudiante- sin embargo no respeto su forma de pensar cuando es distinta a la mía (Solange).

La situación anterior grafica muy bien la tensión expresada entre valorar la autonomía de los estudiantes y sus maneras de pensar, y respetar la opinión que poseen. No siempre resultó sencillo despojarse de las propias maneras de pensar y valores para promover la reflexión. Sin embargo, cuando lo logramos, reconocimos que ello ocurría porque éramos capaces de respetar suspender el juicio y dejarlos explorar sus posibilidades de manera más abierta y confiada.

Por otro lado, reconocimos que la reflexión no puede esperar respuestas rápidas y que incluso, en ocasiones, es un proceso que se sigue desarrollando fuera del espacio formativo de la práctica:

Creo que eso es una construcción. Yo creo que no depende de nosotras [...], pero creo que las mayores herramientas van a salir de ellos mismos, porque ellos están en la situación [...] Entonces nosotros ayudamos a 
mirar, en la medida que nosotros también podemos ver aquello, nosotros no estamos en la sala todos los días [...] hay cosas que a nosotros se nos escapan (Mariluz).

Sabemos que el tiempo de cada sesión de trabajo es acotado, como de igual modo, la duración de los cursos, por lo que aun cuando estábamos conscientes de que una exploración más abierta podría ser muy provechosa para la reflexión, teníamos ciertos límites. De esta manera, pudimos observar que la reflexión debía permitirles reconocer un conflicto de algún tipo para -desde ese lugar- permitir al estudiante introducirse en nuevas comprensiones:

La tarea más importante del tutor no es salvar a los estudiantes de sus errores, sino hacerles preguntas que los Ileven a cuestionarse y reconstruir sus ideas frente a las distintas dimensiones del ser profesor. Esta identidad que se construye de forma conjunta, entre la escuela, el futuro docente y el tutor [...] modelar un análisis más sistémico ecológico y no dar los 'tips' de las situaciones escolares que viven los futuros docentes, de manera de generar capacidades que les permitan responder a los distintos desafíos que implica ser profesor (Solange).

De esta forma, avanzar hacia la reflexión fue entendido como un proceso de brindar ayudas para comprender las situaciones problemáticas que cada uno de nuestros estudiantes iba enfrentando, lo que implicaba una manera distinta de retroalimentarlos, de proveer preguntas que permitan al estudiante analizar su práctica. Había que tener precaución, entonces, de no emitir juicios a sus desempeños y soluciones a las dificultades en que se encuentran:

[...] Cuando una se hace consciente de lo que está haciendo se produce un cambio en el aula [...]. Esto es inmediato y nuestros alumnos lo sintieron. En todos los casos cuando nosotros transparentamos a nuestros alumnos que estábamos reflexionando sobre nuestra práctica. [...] un alumno me dijo que notaba el cambio [frente a una situación problemática del aula, normalmente yo diría: ] 'en estos casos tú puedes hacer esto o lo otro' y el cambio es 'por qué decidiste esto' [... Había un alumno molestando en clases, entonces la pregunta normal hubiese sido 'por qué no le llamaste la atención', pero fue 'tomaste esta determinación, no hiciste nada con el alumno que estaba molestando en clases, ¿por qué? (Mabel).

Junto a ello, la escucha genuina era una forma de entender que el diálogo debía iniciarse a partir de las situaciones que a ellos les tensionaban y no desde marcos previamente delimitados o rígidamente estructurados, aun cuando en ocasiones tengamos un cierto temario que abordar:

[...] una vez conversamos acerca de que queríamos que reflexionaran como nosotras queremos... y eso fue algo que a mí, te juro, que me cambió el paradigma. Nosotros teníamos un taller de reflexión que era temático, poníamos textos. De hecho, teníamos un listado de textos enorme para hablar 
de comunidad de aprendizaje, de reflexión, de clima de aula, de experiencias escolar, etc. Iban pauteados y por lo tanto, teníamos que reflexionar sobre esos temas [...] que no está mal, pero que no tomaba la experiencia de los estudiantes para reflexionar. $Y$ tal vez a eso ponerle marco teórico y no al revés, porque insisto, ahí no estaba la experiencia de los futuros profesores, éramos nosotras nuevamente, lo que nosotras queríamos que reflexionaran [...] en el fondo, porque no era tan obvio (Mariluz).

Considerando que el objetivo es que el estudiante reflexione y fundamente su acción pedagógica, tomamos conciencia de la importancia de que la tutora suspenda su juicio y dialogue con los estudiantes para conocer los argumentos que sustentan sus decisiones. Si bien somos conscientes de que el proceso reflexivo es personal y requiere mecanismos diferenciados de acompañamiento, en el contexto de las distintas experiencias y espacios que poseen los estudiantes para el ejercicio permanente de reflexión acerca de la práctica, no obstante, reconocemos que es un proceso complejo que requiere constante diálogo, apertura y aceptación del otro por parte del tutor. En este contexto, se valoró la creación de instancias colectivas para la reflexión de las propias prácticas, pues estas permiten mirar aquello que muchas veces no se es consciente o no se quiere "ver".

\section{Algunas reflexiones finales}

El ejercicio de self-study implicó importantes cambios a nivel individual y colectivo. Si bien inicialmente enunciamos nuestros puntos de partida individual, las conclusiones, al igual como en el apartado de análisis, refieren a la reflexión colectiva. Recogemos aprendizajes comunes alcanzados, tanto desde nuestro rol como formadoras de formadores, como sobre el sentido mismo de la formación inicial docente. Presentamos las más relevantes.

1. Ya había señalado Rodríguez (2006) que la/el profesor reflexiona -entre otras cosas- sobre las relaciones de poder dentro de la escuela y sus manifestaciones en el currículum, por lo que no es extraño que adquiera un compromiso político en favor de los oprimidos. En nuestro caso, nuestras experiencias como profesoras de aula escolar, así como la propia historia personal han configurado también nuestro ideal de profesor/a y con ello, es evidente que nuestras creencias y concepciones también intencionan lo que esperamos de nuestros estudiantes (Pozo, Scheuer, Mateos \& Pérez Echevarría, 2006). Este aprendizaje nos llevó a poner nuestros esquemas a un lado para permitirles a los estudiantes reflexionar más libremente, favoreciendo la búsqueda de sus propias formas de leer el rol social que tiene la profesión docente. 
En el proceso de tratar de elaborar una definición conjunta nos dimos cuenta que las respuestas eran muy variadas y en ocasiones estábamos esperando un nivel de desempeño que incluso mucho profesores en ejercicio no alcanzan jamás. Consensuamos sobre la importancia de instalar un habitus reflexivo (Perrenoud, 2007) que le permitiera al estudiantado resolver los problemas de su práctica en cualquier momento de su carrera profesional. Ello significó asumir la necesidad de extender la discusión acerca de los límites y alcances de la formación inicial docente con el resto de los académicos de nuestras instituciones. Del mismo modo, implicó que, considerando que el tiempo en los espacios de reflexión con ellos es escaso para la cantidad de problemáticas que los estudiantes traen, aprendimos a organizar mejor la actividad conjunta (Clarà, Mauri, Colomina \& Onrubia, 2019) en relación a los propósitos de cada espacio formativo.

2. Nuestra dinámica de trabajo colaborativo nos hizo vivenciar la gran importancia de contar con un espacio sano de intercambio de experiencias. Descubrimos que la creación de condiciones socioafectivas adecuadas, un espacio protegido y de diálogo honesto y genuino, la valoración del otro y la escucha atenta (Schuck \& Russell, 2016), eran elementos trascendentales para nuestra comunidad profesional de aprendizaje. Este modelo de diálogo se convirtió en referente para el trabajo con nuestros estudiantes, con lo cual comenzamos paulatinamente a vincularnos de manera más consciente con ellos, mediante una escucha receptiva, atenta y consciente, conocedoras de que los procesos reflexivos solo se desarrollarían cuando los estudiantes fueran puestos en tensión con las preguntas adecuadas.

3. En relación a este último punto, al analizar nuestro trabajo notamos que la reflexión dentro del espacio de las prácticas podía tomar distintas rutas. En todos los casos la reflexión era sobre la acción (Schön, 1998), ya fuera propia de cada estudiante como de sus compañeros/as. En este sentido, convenimos en que dejar fluir la reflexión a partir de las preocupaciones que los estudiantes traían a la clase o taller era necesario para dotar de significado el trabajo y no coartar el análisis. Para ello se requería elaborar buenas preguntas que actuaran como gatilladoras o generadoras de conflicto para profundizar el análisis (Gelfuso, 2016). De esta forma, el mayor desafío se instala en poder equilibrar mejor un estilo de conducción: más abierto en el inicio de la discusión, para favorecer que emerjan las problemáticas de interés y más directivo hacia el final, para hacer converger las voces participantes (Liu, 2017). 
4. Una de las particularidades del espacio de prácticas es que resulta muy demandante y estresante en términos personales y profesionales, porque en el aula se conjugan distintos elementos, incluidos los propios sentimientos, creencias y actitudes (Rodríguez, 2006). Como profesoras en los espacios de prácticas, hemos sido testigos de diversas problemáticas que -en ocasiones- exceden al quehacer profesional. Para nosotras, el sentido de reflexionar sobre la práctica está muy ligado a ayudar a los estudiantes a descubrirse y convertirse en profesor/a desde el ejercicio permanente del auto-cuestionamiento de su práctica, el descubrimiento de sus propias fortalezas y dificultades, el asumir el error como un espacio para aprender y el trabajo de su propia autonomía. En este contexto, nos preguntamos dónde estaba el límite entre la supervisión de prácticas y la terapia (Korthagen $\&$ Vasalos, 2005). Si bien no tuvimos acuerdo en este punto, el aprendizaje fue que para definirlo, era importante que cada una estableciera los límites respecto de las finalidades de dicho espacio formativo y de las propias capacidades profesionales para abordar problemáticas de identidad profesional.

En síntesis, en relación a las lecciones sobre este self-study quisiéramos señalar la importancia de respetar las propias creencias de los estudiantes, aunque a veces no las compartiéramos, no imponer las nuestras; que la reflexión es un proceso largo e inacabado que requiere de la escucha activa y amorosa por parte de las académicas; y que la organización de la actividad conjunta guiará el tipo de resultado que queremos, incluyendo la posibilidad de que se abra a una discusión mucho más privada y personal.

Somos conscientes de que la reflexión acerca de la propia práctica no implica necesariamente cambios en la misma. Para ello, resulta fundamental implicarse en el cambio. Demanda, por tanto, un acto de valentía para enfrentar la incertidumbre de lo nuevo, pues lo que esperamos no depende solo de nosotras. Fue necesario dejar el control y dar paso a un proceso de aprendizaje distribuido, significativo y complejo, así como poner especial cuidado en comprender las actuaciones y comportamientos de los estudiantes a partir de sus propias historias de vida, representaciones y creencias en torno a la práctica educativa: el cómo se enseña y se aprende.

Sabemos a su vez que la enseñanza de la reflexión nos sitúa del mismo modo a las formadoras como modelos de reflexión (Russell \& Martin, 2017). Creemos que la metodología de self-study debiera potenciarse al interior de las instituciones de educación superior como un espacio para dialogar y co-construir para la mejora de los aprendizajes de los y las estudiantes. Los/as profesores/as universitarios/as debemos aprender a examinar 
con mayor detalle nuestro quehacer, identificar las claves de nuestra acción y las necesidades de nuestros/as estudiantes, atendiendo de manera sensible a sus preocupaciones y elaborando las preguntas esenciales que les abran la posibilidad de nuevos aprendizajes y crecimiento profesional, tanto individual como colectivo.

Valoramos profundamente la instancia de aprendizaje colaborativo derivada de este self-study. En el ambiente universitario no es frecuente contar con espacios para poner sobre la mesa los distintos conocimientos derivados de nuestro propio quehacer (Clarà \& Mauri, 2010) y este tipo de metodología de investigación lo permite. Las experiencias compartidas nos permitieron nutrir el repertorio de actuaciones (Schön, 1998) con lo cual hemos podido recoger en particular diferentes aprendizajes asociados a las mejores estrategias de acompañamiento para enseñar a reflexionar en cada contexto.

Por lo anterior, creemos relevante que al interior del cuerpo docente de las universidades se releve el valor a la "autoridad de la experiencia", pues tanto profesores/as como estudiantes necesitamos aprender de la práctica y visualizar dicha experiencia como una fuente de conocimiento válido (Russell \& Martin, 2017). Lo anterior nos desafía a las y los formadores de formadores a salir de una racionalidad técnica y valoración del conocimiento académico sobre el experiencial en los contextos de prácticas y reflexión sobre la práctica y abrir espacios al interior del cuerpo docente para que se instalen discursos y racionalidades distintas a las atribuidas actualmente, y a veces impuestas, en las aulas universitarias.

Finalmente, creemos que introducirse verdaderamente en la práctica reflexiva, acompañado de los pares, no siempre fue una tarea dulce, pues implicó salir de nuestras zonas de comodidad, develar nuestras tensiones, aventurarnos y atrevernos a hacer cosas distintas, incursionar en otras maneras de relacionarnos y acercarnos a esa relación dialógica tan pretendida por todos. Nos preguntamos, también a partir de la experiencia vivida, si efectivamente los amigos críticos requieren de ciertas cualidades, experiencia y condiciones personales que le permitan realizar esta tarea, como de igual modo, de condiciones institucionales que provean espacios colectivos de reflexión para la mejora de la práctica.

\section{Referencias bibliográficas}

Blanch, S. \& Corcelles, M. (2014). Sistemas de formación basados en la cooperación entre docentes. En C. Monereo (coord). Enseñando a enseñar en la universidad. La formación del profesorado basada en incidentes críticos. Octaedro: Barcelona 
Castellanos, S. \& Yaya, R. (2013). La reflexión docente y la construcción de conocimiento: una experiencia desde la práctica. Sinéctica, 41, 2-18.

Clarà, M. \& Mauri, T. (2010). El conocimiento práctico. Cuatro conceptualizaciones constructivistas de las relaciones entre conocimiento teórico y práctica educativa. Infancia y Aprendizaje, 33(2), 131-141.

Clarà, M., Mauri, T., Colomina, R. \& Onrubia, J. (2019). Supporting collaborative reflection in teacher education: a case study. European Journal of Teacher Education, 42(2), 175-191. https://doi.org/10.1080/02619768.2019.1576626

Cornejo, J. (2016). El Self-Study de la práctica de los formadores de futuros profesores: bases teóricas, características y modalidades metodológicas. En T. Russell, R. Fuentealba y C. Hirmas (Comp.), Formadores de formadores, descubriendo la propia voz a través del Self-Study (pp. 25-65). Santiago: OEI.

Fenstermacher, G. D. (1987). Prologue to My Critics. Educational Theory, 37(4), 357-360. https://doi.org/10.1111/j.1741-5446.1987.00357.x

Gelfuso, A. (2016). A framework for facilitating video-mediated reflection: Supporting preservice teachers as they create "warranted assertabilities" about literacy teaching and learning. Teaching and Teacher Education, 58, 68-79.https://doi.org/10.1016/j. tate.2016.04.003

Guerra, P. (2009). Revisión de experiencia de reflexión en la formación inicial de docentes. Revista Estudios Pedagógicos, 35(2), 243-260. https://doi.org/10.4067/S0718$\underline{07052009000200014}$

Korthagen, F. \& Vasalos, A. (2005). Levels in reflection: Core reflection as a means to enhance professional growth. Teachers and Teaching, 11(1), 47-71. https://doi. org/10.1080/1354060042000337093

Liu, K. (2017). Creating a dialogic space for prospective teacher critical reflection and transformative learning. Reflective Practice, 18(6), 805-820. https://doi.org/10.1 $\underline{080 / 14623943.2017 .1361919}$

Monereo, C (2014). Salir de la crisis. Sistemas de formación basados en el análisis de incidentes críticos. En Monereo, C (coord.). Enseñando a enseñar en la universidad. La formación del profesorado basada en incidentes críticos. Octaedro: Barcelona.

Moreno, T (2006). La colaboración y colegialidad docente en la universidad: del discurso a la realidad. Perfiles Educativos, 112, 98-130

Perrenoud, P. (2007). Desarrollar la práctica reflexiva en el oficio de enseñar. Barcelona: Grao.

Pozo, J., Scheuer, N., Mateos, M. \& Pérez Echevarría, M. (2006). Las teorías implícitas sobre el aprendizaje y la enseñanza. En J. Pozo, N. Scheuer, M. Pérez Echevarría, M. Mateos \& M. de la Cruz (Eds.), Nuevas formas de pensar la enseñanza y el aprendizaje. Las concepciones de profesores y alumnos (pp. 29-54). Barcelona: Graò.

Rodríguez, A. (2006). Análisis y Mejora de la Propia Enseñanza. Contexto e Educação, 21(76), 127-150.

Russell, T. \& Martin, A. K. (2017). Teacher education needs an epistemology of practice. In J. Mena et al. (Eds.), Search and research: Teacher education for contemporary contexts. (pp. 111-118). Salamanca: Ediciones Universidad Salamanca

Schön, D. (1998). El Profesional reflexivo: cómo piensan los profesionales cuando actúan. Barcelona: Paidós Ibérica.

Schuck, S. \& Russell, T (2016). Self-Study, Amistad crítica y las complejidades en la formación de profesores. En T. Russell, R. Fuentealbay C. Hirmas (Comp.), Formadores de formadores, descubriendo la propia voz a través del Self-Study (pp. 117-132). Santiago: OEI 